\title{
Correction to: Single-incision laparoscopic surgery (SILS) for the treatment of ileocolonic Crohn's disease: a propensity score-matched analysis
}

\author{
Valerio Celentano ${ }^{1,2,3}$ (D) Gianluca Pellino ${ }^{4,5}$ - Matteo Rottoli ${ }^{6}$. Francesco Colombo ${ }^{7}$. Gianluca Sampietro ${ }^{8}$. \\ Antonino Spinelli $i^{9,10}$. Francesco Selvaggi ${ }^{4}$ - on behalf of the Italian Society of Colorectal Surgery SICCR \\ Published online: 8 January 2021 \\ (C) Springer-Verlag GmbH Germany, part of Springer Nature 2021
}

\section{Correction to: International Journal of Colorectal Disease https://doi.org/10.1007/s00384-020-03821-6}

All authors and affiliations of the SICCR Current status of Crohn's disease surgery collaborative are listed in Appendix 1 and Specification of authors' contribution is detailed in Appendix 2 of the original version of the above article however, the names were not cited nor reflected online. Thus, the names are now presented and cited correctly. All authors to be indexed on Pubmed.

The affiliation of Francesco Colombo a co-author of the above article is affiliated to "General Surgery Unit, Department of Biomedical and Clinical Sciences "L. Sacco". University of Milan, AAST Fatebenefratelli Sacco, Milan, Italy" instead of "Division of General and HPB Surgery, Department of Surgery, ASST Rhodense - Rho Memorial Hospital, 20017 Rho, Milan, Italy". The original article has been corrected.

Publisher's note Springer Nature remains neutral with regard to jurisdictional claims in published maps and institutional affiliations.

The online version of the original article can be found at https://doi.org/ 10.1007/s00384-020-03821-6

Valerio Celentano

valeriocelentano@yahoo.it

1 Portsmouth Hospitals NHS Trust, Portsmouth, UK

2 University of Portsmouth, Portsmouth, UK

3 Department of Surgery and Cancer, Imperial College, London, UK

4 Department of Advanced Medical and Surgical Sciences, Universita' degli Studi della Campania Luigi Vanvitelli, Naples, Italy

5 Colorectal Surgery, Vall d'Hebron University Hospital, Barcelona, Spain
6 Surgery of the Alimentary Tract, Department of Medical and Surgical Sciences, Sant'Orsola Hospital, Alma Mater Studiorum University of Bologna, Bologna, Italy

7 General Surgery Unit, Department of Biomedical and Clinical Sciences "L. Sacco", University of Milan, AAST Fatebenefratelli Sacco, Milan, Italy

8 Division of General and HPB Surgery, Department of Surgery, ASST Rhodense - Rho Memorial Hospital, 20017 Rho, Milan, Italy

9 Division of Colon and Rectal Surgery, Humanitas Clinical and Research Center IRCCS, Via Manzoni 56, 20089 Rozzano, Milan, Italy

10 Department of Biomedical Sciences, Humanitas University, Via Rita Levi Montalcini 4, 20090 Pieve Emanuele, Milan, Italy 